
Pathophysiology of Haemostasis and Thrombosis

\title{
The Evidence behind Inhibitor Treatment with Porcine Factor VIII
}

\author{
Christine A. Lee \\ The Haemophilia Centre and Haemostasis Unit, Royal Free Hospital NHS Trust, London, UK
}

\section{Key Words}

Porcine FVIII - Inhibitor • Pharmacokinetics · Hybrid FVIII • Acquired

\begin{abstract}
Factor VIII auto- and alloantibodies neutralise porcine factor VIII to a lesser extent than factor VIII of human origin. The reduced reactivity of the porcine molecule, predominantly due to sequence variation in the $\mathrm{A} 2$ and $\mathrm{C} 2$ domains, has been the rationale for using porcine factor VIII to secure haemostasis for patients with factor VIII inhibitors. Porcine factor VIII has been shown to provide effective haemostatic control particularly for patients with intermediate inhibitor titres with limited porcine cross-reactivity. Small studies have indicated porcine factor VIII can be associated with desensitisation of some factor VIII inhibitor patients. Porcine factor VIII has been shown to produce mild platelet agglutination, an effect that may enhance its efficacy. Adverse reactions are dose-related and do not preclude safe and effective longterm home use for the subgroup of inhibitor patients with modest or absent anamnestic response. Efforts to secure source plasma free of viral markers, particularly porcine parvovirus, have limited the supply of this therapeutic product.
\end{abstract}

Copyright $\odot 2002$ S. Karger AG, Base

\section{Introduction}

Porcine factor (F)VIII (Hyate: $\left.\mathrm{C}^{\circledR}\right)$ is a second-generation FVIII concentrate of porcine plasma origin, fractionated using a polyelectrolyte resin. Although there is no specific viral attenuation step during the production of this concentrate, a four-cell line general viral screen and a polymerase chain reaction source plasma screen for porcine parvovirus are performed. Porcine FVIII has not been shown to transmit viral disease.

The first published clinical experience with polyelectrolyte fractionated porcine FVIII concentrate in the treatment of haemophilic patients with antibodies to FVIII was reported in 1984 [1]. It is of interest that the first patient to be treated in 1980 is still using this treatment as home therapy. This review will present the published evidence base for the clinical use of porcine FVIII, together with a brief summary of research development of porcine-human FVIII hybrid constructs.

\section{Porcine FVIII as an Inhibitor Bypassing Agent}

Porcine FVIII has been effective in treating acute bleeding in patients with congenital or acquired haemophilia. The first publication gave a detailed description of 45 treatment courses in eight inhibitor patients, a total of 297 infusions [1]. Where no cross-reactivity to porcine

Prof. Christine Lee, Centre Directo

Royal Free Hospital, Pond Street

London, NW3 2QG (UK)

Tel. +44 2077830 2238, Fax +44 2077830 2468, E-Mail christine.lee@rfh.nthames.nhs.uk
Fax + 41613061234

E-Mail karger@karger.ch

www. karger.com
Accessible online at:

www. karger.com/journals/pht 
FVIII was detectable, the mean increment in plasma FVIII was $1.29 \mathrm{IU} / \mathrm{dl}$ per IU/kg of porcine factor VIII administered. With increasing porcine FVIII cross-reactivity, factor VIII recovery progressively declined. Where post-infusion FVIII recovery was detectable, clinical response was good and conventional FVIII:C assay could be used to guide dosage. The study concluded that the product was an effective haemostatic agent for patients with FVIII inhibitors, particularly those with intermediate level anti-human FVIII titres where cross-reactivity to porcine FVIII was limited. A study from the United States described 45 life or limb threatening bleeding episodes in 38 patients with high-responding inhibitors [2]. A total of 437 infusions were administered, with a good or excellent response recorded in $71 \%$ of bleeds. Adverse reactions occurred in 17 treatment episodes, and responded to antihistamines with or without hydrocortisone. The most common infused dose was $50 \mathrm{IU} / \mathrm{kg}$.

Porcine FVIII concentrate has an advantage over human-derived FVIII in patients with congenital haemophilia and inhibitors since the antibodies have a median of only $15 \%$ cross-reactivity with porcine FVIII [3]. In addition, patients with acquired haemophilia usually respond well to porcine FVIII, particularly when they have low or absent anti-porcine inhibitor titres. In general, patients with inhibitor titres of $<20 \mathrm{BU} / \mathrm{ml}$ have a better response rate than those with higher titres [1]. Some patients with congenital haemophilia maintain low antiporcine inhibitor titres over long term use and can therefore be treated routinely at home over many years. It is possible that the platelet agglutinating effect of porcine von Willebrand factor (vWF) may contribute to the response in patients with high titre inhibitors. An anamnestic response has been reported to occur in 25$35 \%$ of treatments with porcine FVIII, but this risk is relatively low among patients with acquired haemophilia. Most patients with congenital haemophilia can be treated for serious bleeding before the development of clinically significant anti-porcine antibodies.

\section{Home Therapy with Porcine FVIII}

Porcine FVIII has been used safely and effectively in the home therapy setting for extended periods. An observational study described the experience of five haemophilia A patients with high-titre, high-responding FVIII alloantibodies who received porcine FVIII for periods of up to 8.5 years [4]. Specific anti-porcine activity developed in all cases, but was generally transient, with only

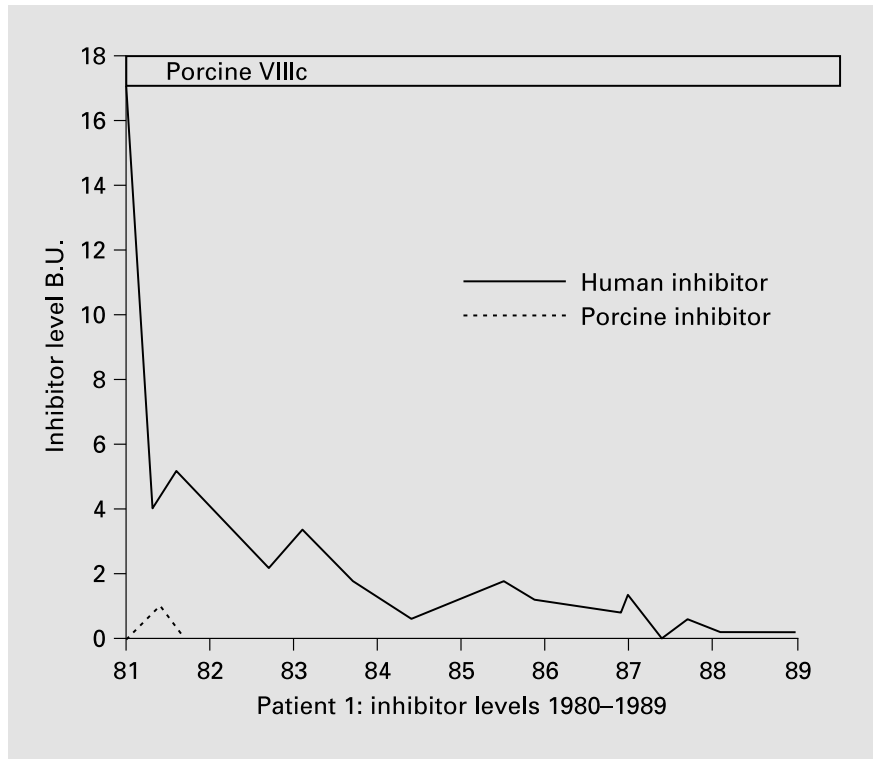

Fig. 1. FVIII desensitisation: inhibitor titres between 1980 and 1989. (From Hay et al, 1997, with permission.)

one patient becoming refractory. Of particular interest, all patients lost their anti-human FVIII activity over time. In three cases, human FVIII was re-introduced successfully, with no recurrence of anti-human FVIII activity over 2-3 years of continued therapy. There were no significant transfusion reactions.

One of the first inhibitor patients to receive porcine FVIII has used the product as required to treat bleeding episodes for over 20 years. In this time, he has undergone five operative procedures, including total knee arthroplasty, using porcine FVIII for haemostatic control. Figure 1 shows his inhibitor titre declining over time and eventually completely disappearing. A notable feature about this patient is that he never developed a significant cross-reactivity to porcine FVIII [5].

An international survey assessed the side effect profile and use of porcine FVIII for regular or home therapy [3]. The study described 154 inhibitor patients who had used over 15 million IU. The median pre-treatment cross reactivity to the porcine FVIII was $15 \%$. There was no evidence of cross reactivity to the porcine product in $27 \%$ of the cases. Figure 2 shows the pre-treatment inhibitor cross reactivity in 137 of the patients. There were very few that had a high cross reactivity and most either had a low cross reactivity or no cross reactivity. Anamnesis was evaluable in 65 patients. Brisk anamnesis, defined as at least a threefold rise in antibody titre following the first treatment,
Pathophysiol Haemost Thromb 2002;32(suppl 1):5-8
Lee 
Fig. 2. Histogram of pre-treatment inhibitor cross-reactivity to porcine FVIII:C in 147 patients. (From Hay et al, 1996, with permission.)

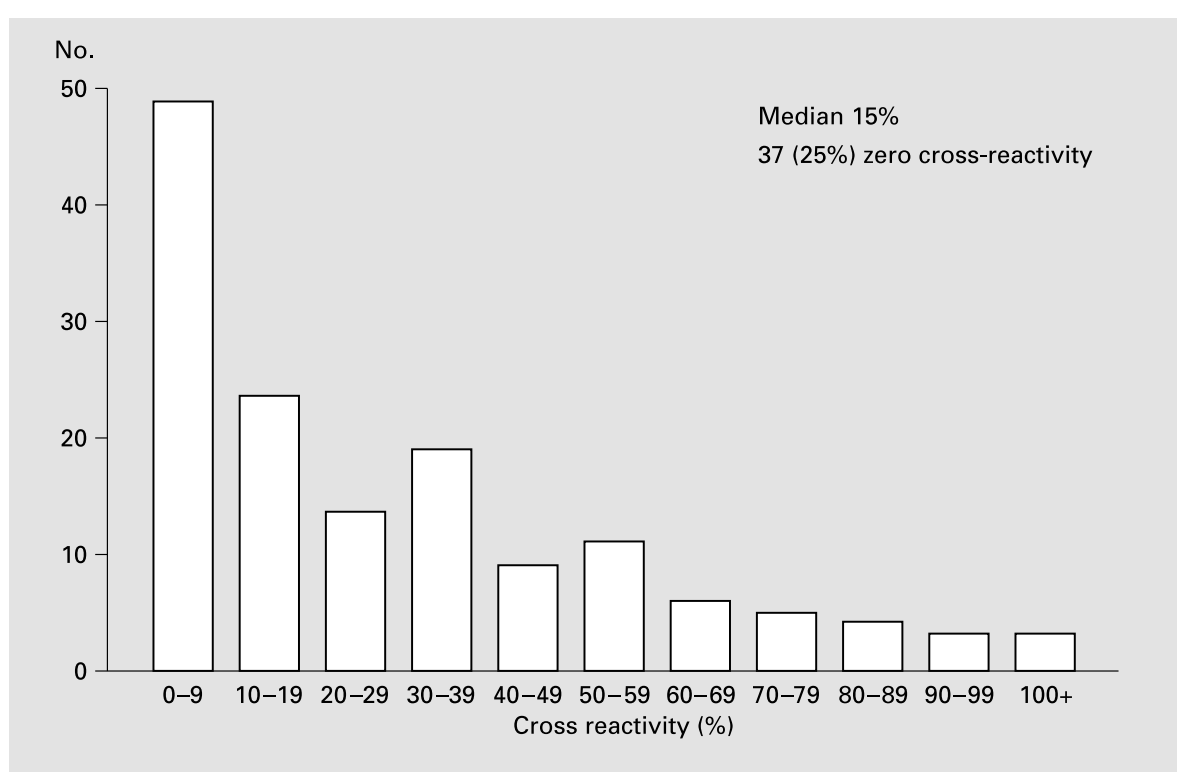

occurred in $31 \%$ of patients. Importantly, $29 \%$ of patients had no anamnestic response following their first treatment and 38\% had no such response following multiple treatments. Transfusion reactions occurred after $0.001 \%$ of home infusions and in $2.3 \%$ of unselected inpatient infusions, with incidence of adverse reactions showing a positive association with dosage.

A post-infusion fall in platelet count is common after administration of porcine FVIII and is due to reversible agglutination by porcine von Willebrand factor. The international survey collected platelet count data around 175 porcine FVIII infusions for 57 individuals. After a median porcine FVIII dose of $96 \mathrm{U} / \mathrm{kg}$, the median fall in platelet count was $34 \times 10^{9} / 1$, occurring at about $30 \mathrm{~min}-$ utes after the infusion. The platelet count reduction was clinically insignificant in all cases [3].

A survey of acquired haemophilia in Europe and the US assessed 74 bleeding episodes in 65 patients [6]. At presentation the median inhibitor titre against human FVIII was $38 \mathrm{BU} / \mathrm{ml}$ and against porcine FVIII was $1 \mathrm{BU} /$ $\mathrm{ml}$. The average initial dose of porcine FVIII was $84 \mathrm{IU} /$ $\mathrm{kg}$ resulting in a mean increase in plasma FVIII:C of $0.85 \mathrm{IU} / \mathrm{ml}$. Haemostatic response was rated as good or excellent in $78 \%$ of cases. The mean duration of therapy was 8.5 days, sufficient in most cases to control bleeding episodes whilst the immune suppression therapy was taking effect.

The half-life of porcine FVIII has considerable interindividual variability and has been reported to range from
8 to 24 hours. In addition, FVIII levels measured using the chromogenic assay are on average 50\% lower than those measured by the one-stage method [7]. The mechanism for this observation has not been clarified. Vial potency of porcine FVIII is assigned by the chromogenic method.

\section{Human/Porcine Hybrid FVIII}

Inhibitory antibodies are directed mainly at epitopes in the A2 (residues 373-740), C2 (residues 2173-2332) or A3 domains of the factor VIII molecule. There is evidence that antibodies that recognise epitopes in the A2 domain bind the tenase complex and thereby prevent activation of FX [8]. Antibodies binding to the $\mathrm{C} 2$ domain block the interaction of FVIII with phospholipid and may also impair FVIII binding of vWF [9]. Antibodies recognising the A3 domain may inhibit the FVIIIa-FIXa interaction. Recently, recombinant human-porcine hybrid FVIII constructs have been developed with porcine substitutions of the sectors of the human A2 and $\mathrm{C} 2$ domains that are known to be more immunogenic [10]. The resultant hybrid human/porcine FVIII molecules have been shown to produce full procoagulant activity, with reduced recognition by neutralising factor VIII antibodies. These constructs may not only be useful as treatment of bleeding in inhibitor patients but may also be less immunogenic than wild-type FVIII for normal use.
The Evidence behind Inhibitor Treatment with Porcine Factor VIII
Pathophysiol Haemost Thromb 2002;32(suppl 1):5-8 


\section{Summary}

There is a strong evidence base for the use of porcine FVIII, in both congenital haemophilia with FVIII alloantibodies and in acquired haemophilia. It is effective in the subset of inhibitor patients who demonstrate low porcine cross-reactivity. In this group, modest doses - in the region of 30-40 IU/ $\mathrm{kg}$ - have been used to effectively control bleeding for long term use in the home environment. There are examples where induction of immune tolerance to human FVIII has occurred with long-term use of the porcine product. The prime issue restricting supply of porcine FVIII is the number of pigs infected with porcine parvovirus. While there is no evidence to suggest porcine parvovirus is pathogenic to humans, any infected porcine plasma pools are rejected. This has resulted in rejection of positive pools in $50-80 \%$ of cases and has led to extreme shortages in supply of useable plasma.

\section{References}

1 Kernoff PB, Thomas ND, Lilley PA, Matthews KB, Goldman E, Tuddenham EG: Clinical experience with polyelectrolyte-fractionated porcine factor VIII concentrate in the treatment of hemophiliacs with antibodies to factor VIII. Blood 1984;663:31-41.

2 Brettler DB, Forsberg AD, Levine PH, Aledort LM, Hilgartner MW, Kasper CK, Lusher JM, McMillan C, Roberts H: The use of porcine factor VIII concentrate (Hyate:C) in the treatment of patients with inhibitor antibodies to factor VIII. A multicenter US experience. Arch Intern Med 1989;149:1381-1385.

3 Hay CR, Lozier JN, Lee CA, Laffan M, Tradati F, Santagostino E, Ciavarella N, Schiavoni M, Fukui H, Yoshioka A, Tietel J, Mannucci PM, Kasper CK: Safety profile of porcine factor VIII and its use as hospital and home-therapy for patients with haemophilia A and inhibitors: The results of an international survey. Thromb Haemost 1996;75:25-29.
4 Hay CR, Laurian Y, Verroust F, Preston FE, Kernoff PB: Induction of immune tolerance in patients with hemophilia $\mathrm{A}$ and inhibitors treated with porcine VIIIC by home therapy. Blood 1990;76:882-886.

5 Vyas P, Pasi KJ, Lee CA: Successful long-term treatment with porcine factor VIII of a patient with haemophilia A and an inhibitor to factor VIII. Haemophilia 1996;2:240-243.

6 Morrison AE, Ludlam CA, Kessler C: Use of porcine factor VIII in the treatment of patients with acquired hemophilia. Blood 1993;81: 1513-1520.

7 Hermans C, Owens D, Longo G, Morfini M, Lee C.A: Single-dose pharmacokinetics of porcine factor VIII (Hyate C). Thromb Haemost 2002;87:352-353.
8 Lollar P, Parker ET, Curtis JE, Helgerson SL, Hoyer LW, Scott ME, Scandella D: Inhibition of human factor VIIIa by anti-A2 subunit antibodies. J Clin Invest 1994;93:2497-2504.

9 Arai, M, Scandella D, Hoyer LW: Molecular basis of factor VIII inhibition by human antibodies. Antibodies that bind to the factor VIII light chain prevent the interaction of factor VIII with phospholipid. J Clin Invest 1989;83: 1978-1984.

10 Lollar P: Analysis of factor VIII inhibitors using hybrid human/porcine factor VIII. Thromb Haemost 1997;78:647-651.

11 Hay CRM, DiMichele D, Rivard G, Rubinger M, Gribble J: Workshop III - porcine factor VIII - continuous infusion, immune tolerance induction and prophylaxis. Haemophilia 1997; 3(suppl 3):24-30. 